\title{
Dynamics in discrete two-dimensional nonlinear Schrödinger equations in the presence of point defects
}

Christiansen, Peter Leth; Gaididei, Yuri Borisovich; Rasmussen, Kim; Mezentsev, Vladimir; Juul Rasmussen, Jens

Published in:

Physical Review B

Link to article, DOI:

10.1103/PhysRevB.54.900

Publication date:

1996

Document Version

Publisher's PDF, also known as Version of record

Link back to DTU Orbit

Citation $(A P A)$ :

Christiansen, P. L., Gaididei, Y. B., Rasmussen, K., Mezentsev, V., \& Juul Rasmussen, J. (1996). Dynamics in discrete two-dimensional nonlinear Schrödinger equations in the presence of point defects. Physical Review $B$, 54(2), 900-912. https://doi.org/10.1103/PhysRevB.54.900

\section{General rights}

Copyright and moral rights for the publications made accessible in the public portal are retained by the authors and/or other copyright owners and it is a condition of accessing publications that users recognise and abide by the legal requirements associated with these rights.

- Users may download and print one copy of any publication from the public portal for the purpose of private study or research.

- You may not further distribute the material or use it for any profit-making activity or commercial gain

- You may freely distribute the URL identifying the publication in the public portal 


\title{
Dynamics in discrete two-dimensional nonlinear Schrödinger equations in the presence of point defects
}

\author{
P. L. Christiansen, Yu. B. Gaididei, ${ }^{*}$ and K. $\emptyset$. Rasmussen \\ Institute of Mathematical Modelling, The Technical University of Denmark, DK-2800 Lyngby, Denmark \\ V. K. Mezentsev ${ }^{\dagger}$ and J. Juul Rasmussen \\ Department of Optics and Fluid Dynamics, Risb National Laboratory, DK-4000 Roskilde, Denmark
}

(Received 5 February 1996)

\begin{abstract}
The dynamics of two-dimensional discrete structures is studied in the framework of the generalized twodimensional discrete nonlinear Schrödinger equation. The nonlinear coupling in the form of the AblowitzLadik nonlinearity and point impurities is taken into account. The stability properties of the stationary solutions are examined. The essential importance of the existence of stable immobile solitons in the two-dimensional dynamics of the traveling pulses is demonstrated. The typical scenario of the two-dimensional quasicollapse of a moving intense pulse represents the formation of standing trapped narrow spikes. The influence of the point impurities on this dynamics is also investigated. [S0163-1829(96)00926-5]
\end{abstract}

\section{INTRODUCTION}

Localization phenomena are widely recognized as a key for understanding the excitation dynamics in many physical contexts such as the theory of light pulse propagation in nonlinear waveguides and charge and energy transport in condensed-matter physics and biophysics. ${ }^{1}$ In the present paper we study the discrete localized stationary states (breathers ${ }^{2}$ and solitons ${ }^{3,4}$ ) and their localization dynamics in the framework of the two-dimensional (2D) generalized discrete nonlinear Schrödinger equations GDNSE's, which in the continuum limit becomes the well-know nonlinear Schrödinger equation (NSE). The GDNSE, we consider, is given by

$$
\begin{gathered}
i \dot{\Psi}_{m, n}+\Omega_{m, n} \Psi_{m, n}+b\left|\Psi_{m, n}\right|^{2} \Psi_{m, n}+\left(\Psi_{m+1, n}+\Psi_{m-1, n}\right. \\
\left.+\Psi_{m, n+1}+\Psi_{m, n-1}\right)\left(J+a\left|\Psi_{m, n}\right|^{2}\right)=0,
\end{gathered}
$$

where $\Psi_{m, n}$ is the complex amplitude of the on-site excitation, $\Omega_{m, n}$ describes the inhomogeneous frequency shift, $b$ measures the intrinsic on-site nonlinearity, and the constants $a$ and $J$ describe the simplest cases of linear and nonlinear coupling between nearest neighbors. The one-dimensional quantized version of this model was considered by Salerno ${ }^{5}$ and recently it was introduced as a model for onedimensional classical lattices by Cai, Bishop, and Grønbech-Jensen. ${ }^{6}$ The GDNSE model represents the superposition of the well-known discrete self-trapping (DST) type of nonlinearity term ${ }^{7}$ and the $2 \mathrm{D}$ form of the Ablowitz-Ladik $^{8}$ (AL) type. If $a=0$, Eq. (1) reduces to the DST equation, which arises in the study of envelope wave propagation in anharmonic lattices in many concrete applications, e.g., in the theory of energy transport in biopolymers ${ }^{9}$ and carrier wave propagation in nonlinear fiber arrays. ${ }^{10}$ The terms that are proportional to $a$ describe the 2D extension of the Ablowitz-Ladik equation, which is integrable for $b=0$ in the one-dimensional case by means of the inverse scattering transform. ${ }^{8}$ Physically speaking, these terms correspond to the nonlinear coupling between nearest neighbors. The properties of the one-dimensional GDNSE were recently analyzed in Ref. 11 and the influence of impurities was considered in Ref. 12. In Ref. 13 the ID GDNSE was derived for an electrical lattice. For the pure DST case the solitons and their stability were studied in Refs. 3 and 4 for cubic nonlinearity in two dimensions and for arbitrary power nonlinearity in the framework of the one-dimensional model. ${ }^{14}$ The soliton shapes and the soliton stability criteria were found and it was also shown that the soliton instability leads to quasicollapse, i.e., fast localization of the initial excitation. The quasicollapse dynamics was investigated for the one-dimensional case in Ref. 15. The defect-soliton interaction in the continuum case was considered in Ref. 16 for one dimension and in Refs. 17 and 18 for two dimensions.

In the present paper we study the soliton solutions and the quasicollapse both in the homogeneous case and in the presence of point defects in the framework of the generalized model equation (1). The paper is organized as follows. In Sec. II we describe the model of the classical twodimensional lattice used in the simulations. In Sec. III the properties of standing solitary solutions are presented. Section IV is devoted to quasicollapse dynamics of standing (Sec. IV A) as well as moving (Sec. IV B) pulses. Also we consider the role of the point defects on the collapse dynamics. Finally Sec. V concludes the paper with a discussion of the results and possible applications.

\section{MODEL}

The starting point of our analysis is the basic equation (1) rewritten in the equivalent form

$$
\begin{aligned}
i \dot{U}_{m, n} & +\omega_{m, n} U_{m, n}-4 U_{m, n}+2(1-\alpha) U_{m, n}\left|U_{m, n}\right|^{2} \\
+ & \left(U_{m+1, n}+U_{m-1, n}+U_{m, n+1}+U_{m, n-1}\right) \\
& \times\left(1+\frac{\alpha}{2}\left|U_{m, n}\right| 2\right)=0,
\end{aligned}
$$




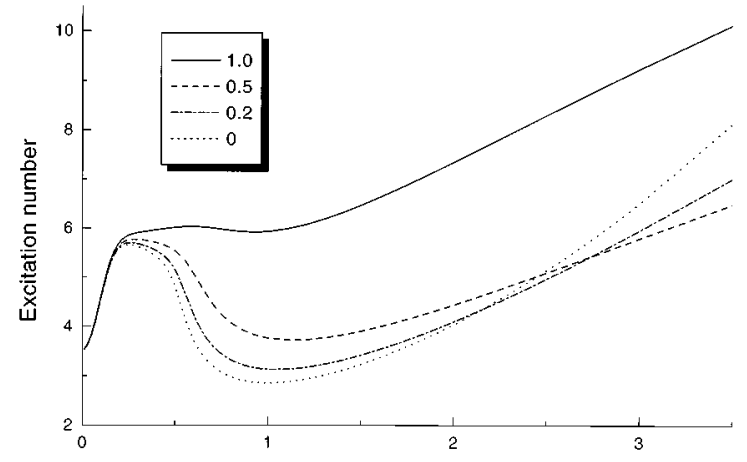

(a)

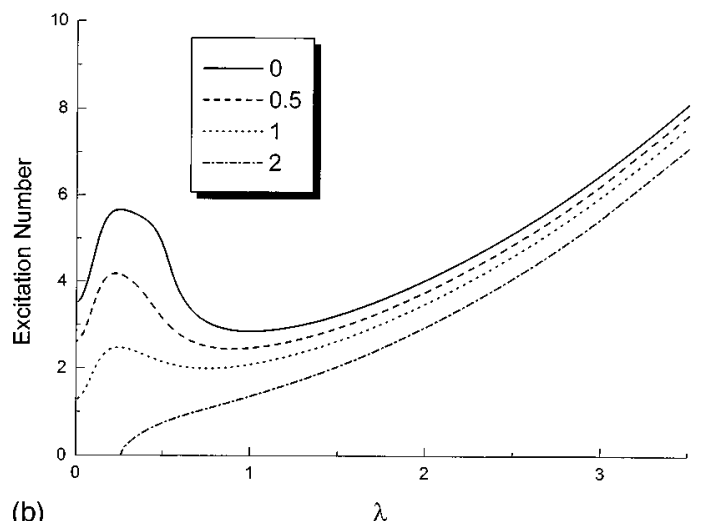

(b)

$\lambda$

FIG. 1. Stationary solutions given by Eqs. (7) and (8). (a) Homogeneous case $\left(\omega_{0}=0\right)$. Excitation number $N$, Eq. (5), versus $\lambda$ for $\alpha=1$ (Ablowitz-Ladik case, solid line), 0.5 (dashed line), 0.2 (dot-dashed line), and 0 (DST, dotted line). (b) Influence of acceptor point defect $\left(\omega_{0}>0, \alpha=0\right)$. Excitation number $N$, Eq. (5), versus spectral parameter $\lambda$ for $\omega_{0}=0$ (homogeneous case, solid line), 0.5 (dashed line), 1 (dotted line), and 2 (dot-dashed line).

where the scale transformations

$$
\begin{gathered}
\Psi=\left(\frac{2 J}{4 a+b}\right)^{1 / 2} e^{i 4 t} U, \quad t=J t^{\prime}, \quad \alpha=\left(1+\frac{b}{4 a}\right)^{-1}, \\
\omega_{m, n}=\Omega_{m, n} / J
\end{gathered}
$$

have been introduced. The factor $\alpha$ shows the relative strength of DST $(\alpha=0)$ and AL $(\alpha=1)$ types of nonlinearities. Equation (2) can be derived from the Hamiltonian

$$
\begin{aligned}
H= & \sum_{m, n}\left(U_{m, n} U_{m, n+1}^{*}+U_{m, n} U_{m+1, n}^{*}+\text { c.c. }\right) \\
& -\frac{2}{\alpha} \sum_{m, n}\left(\frac{4}{\alpha}-\omega_{m, n}\right) \ln \left(1+\frac{\alpha}{2}\left|U_{m, n}\right|^{2}\right) \\
& -4 \frac{\alpha-1}{\alpha} \sum_{m, n}\left|U_{m, n}\right|^{2}
\end{aligned}
$$

accompanied by asymmetric Poisson brackets

$$
\left\{U_{m, n}, U_{m, n}^{*}\right\}=i\left(1+\frac{\alpha}{2}\left|U_{m, n}\right|^{2}\right) \delta_{m, n}, \quad\left\{U_{m, n}, U_{m, n}\right\}=0 .
$$

Then Eq. (2) is expressed as $\dot{U}_{m, n}=\left\{H, U_{m, n}^{*}\right\}$ and it immediately gives the conservation of the Hamiltonian $H$. The generalized model Eq. (2) also conserves the quantity

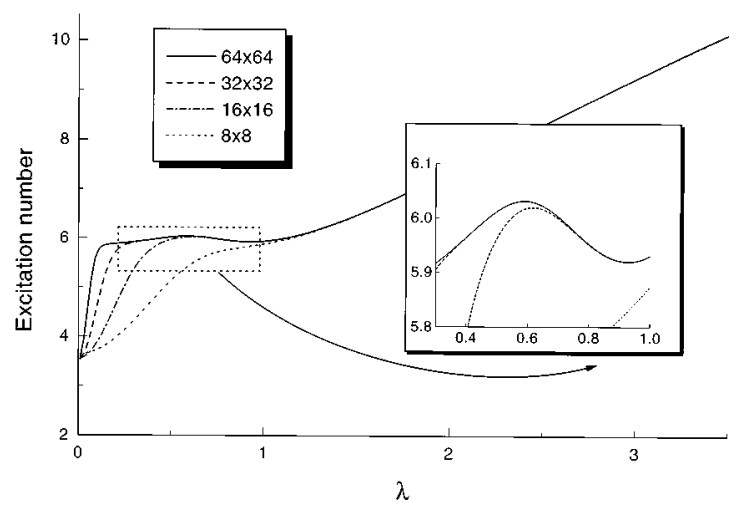

FIG. 2. Stationary solutions given by Eqs. (7) and (8) in the homogeneous Ablowitz-Ladik case $\left(\omega_{0}=0, \alpha=1\right)$. Excitations number $N$, Eq. (5), versus spectral parameter $\lambda$, for grid size $M \times M ; \quad M=64$ (solid line), 32 (dashed line), 16 (dot-dashed line), and 8 (dotted line).

$$
N=\sum_{m, n} \frac{2}{\alpha} \ln \left(1+\frac{\alpha}{2}\left|U_{m, n}\right|^{2}\right),
$$

which we will refer to as the excitation number, noting that in the limit $\alpha \rightarrow 0$ it coincides with the conventional definition $\left(N=\Sigma_{m, n}\left|U_{m, n}\right|^{2}\right)$.

Equation (2) has the continuum limit in the form of a standard cubic nonlinear Schrödinger equation.

$$
\begin{gathered}
i U_{t}+U_{x x}+U_{y y}+2|U|^{2} U=0, \\
x=m L, \quad y=n L, \quad U(x, y)=U_{m, n},
\end{gathered}
$$

where $L$ is the lattice spacing. This model conserves the Hamiltonian $H=\int\left(|\nabla U|^{2}-|U|^{4}\right) d \mathbf{r}$, total momentum $\mathbf{P}=\int\left(i U^{*} \nabla U+\right.$ c.c. $) d \mathbf{r}, \quad$ and excitation number $N=\int|U|^{2} d \mathbf{r}$. It possesses localized stationary and moving 2D soliton solutions. However, they are unstable ${ }^{19}$ and the development of the instability leads to collapse or blowup. In general, the necessary condition for blowup of a localized initial condition is given by the inequality $N>N_{c} \approx 5.86$, where $N_{c}$ is the excitation number of the ground-state soliton solution. Due to the Galilean invariance of the continuum, limit Eq. (6), there is no difference between the evolution for initial conditions corresponding to standing and moving waves. In particular, it means that the collapse dynamics is universal and independent of the movement of the excitation.

\section{STATIONARY SOLUTIONS}

First we consider standing stationary solutions of Eq. (2), i.e., soliton solutions, in the form

$$
U_{m, n}=F_{m, n} \exp (i \omega t),
$$

where both the shape $F_{m, n}$ and the corresponding nonlinear frequency shift $\omega$ are determined by the nonlinear algebraic eigenvalue problem

$$
\begin{aligned}
\varphi_{m, n} \equiv & -\left(\omega-\omega_{m, n}\right) F_{m, n}-4 F_{m, n}+2(1-\alpha)\left|F_{m, n}\right|^{2} F_{m, n} \\
& +\left(F_{m+1, n}+F_{m-1, n}+F_{m, n+1}+F_{m, n-1}\right) \\
& \times\left(1+\frac{\alpha}{2}\left|F_{m, n}\right|^{2}\right)=0 .
\end{aligned}
$$



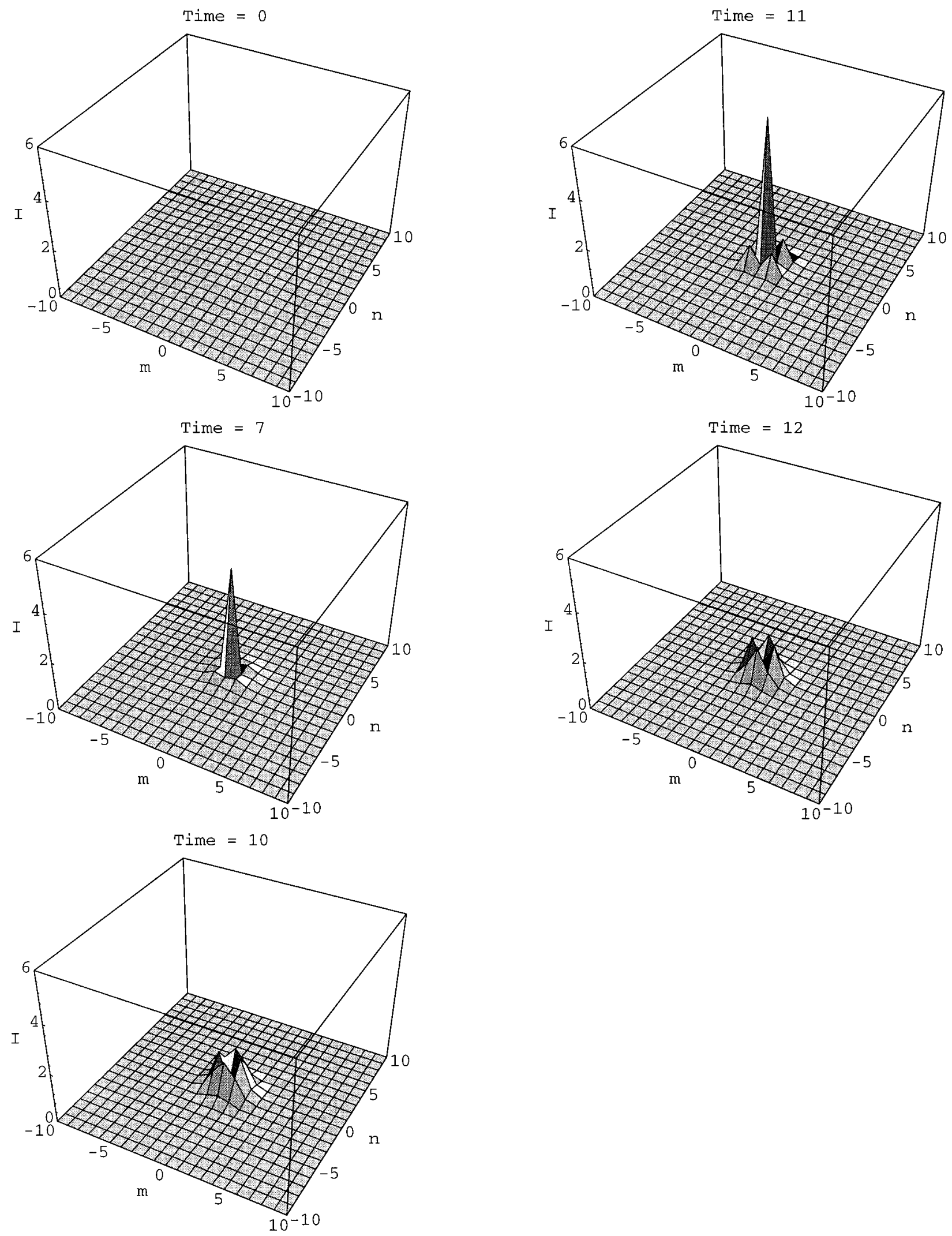

FIG. 3. Dynamics of a standing solution in the homogeneous DST case Eq. (2) with $\omega_{0}=0, \alpha=0$. Initial condition, Eq. (10), with $A_{0}=0.4, m_{0}=n_{0}=8, \kappa_{m}=\kappa_{n}=0$, and $\bar{m}=\bar{n}=0 ; t=0,7,10,11$, and 12 .

The standing 2D soliton solutions defined by (8) in the pure DST case $(\alpha=0)$ were described in Ref. 3. In the present paper we study the effects of both the Ablowitz-Ladik nonlinear coupling and the spatial point defects provided by the additional frequency shift at a particular site: $\omega_{m, n}=\omega_{0} \delta_{m, 0} \delta_{0, n}$ Attracting (repelling) defects correspond to positive (negative) values of $\omega_{0}$. We shall denote the two cases acceptor and donor, respectively. 

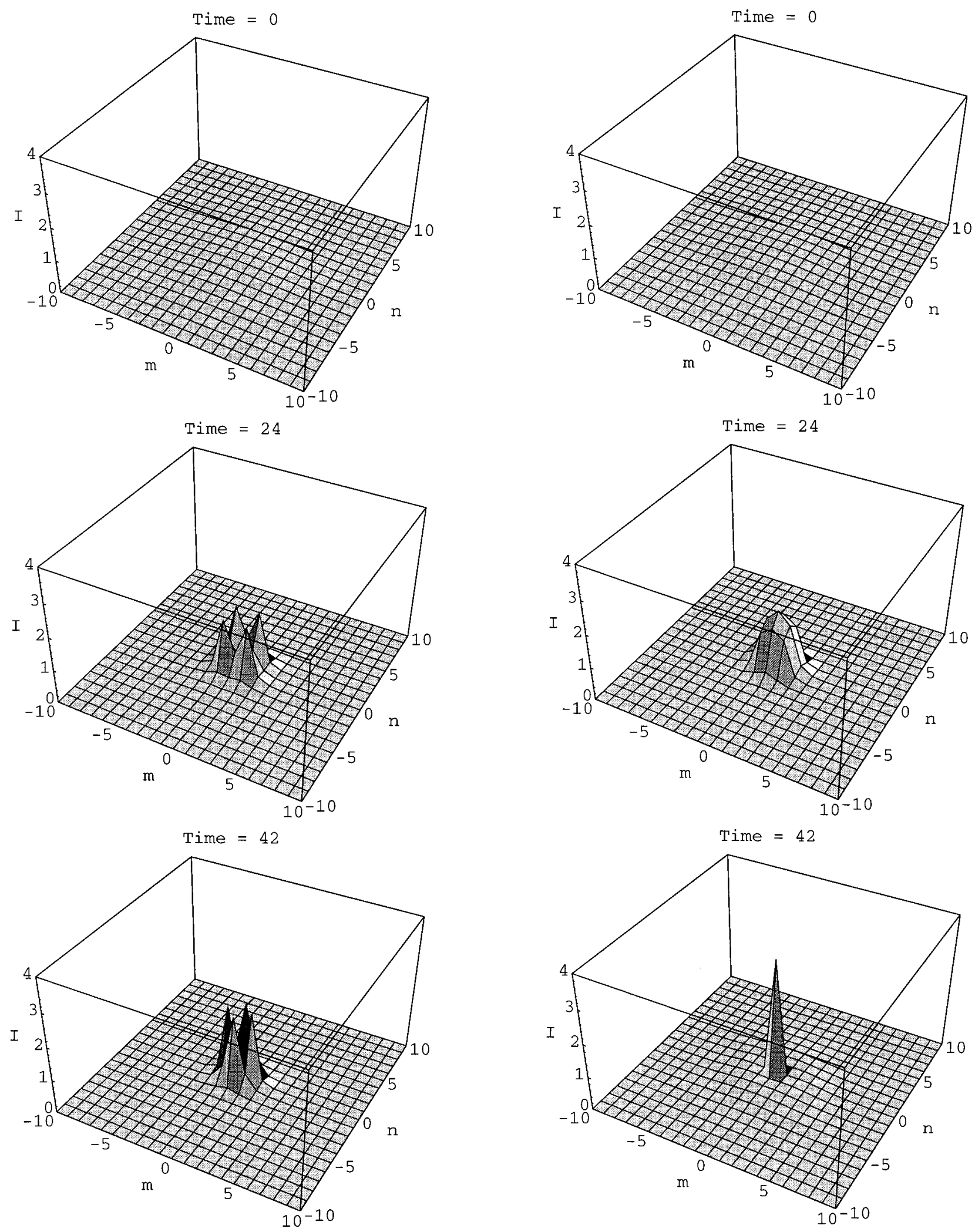

FIG. 4. Dynamics of a standing solution in the inhomogeneous donor DST case Eq. (2) with $\omega_{0}=-4, \alpha=0$. Initial condition, Eq. (10), with $A_{0}=0.4, m_{0}=n_{0}=6, \kappa_{m}=\kappa_{n}=0$, and $\bar{m}=\bar{n}=0 ; t=0$, 24 , and 42 .

The numerical procedure used to solve the nonlinear eigenvalue problem Eq. (8) is a discrete version of the iterative Petviashvili method originally developed to find equilibrium states in plasma physics described in Ref. 20. It can be re-

FIG. 5. Dynamics of a standing solution in the inhomogeneous acceptor DST case Eq. (2) with $\omega_{0}=2, \alpha=0$. Initial condition, Eq. (10), with $A_{0}=0.4, m_{0}=n_{0}=6, \kappa_{m}=\kappa_{n}=0$, and $\bar{m}=\bar{n}=0 ; t=0$, 24 , and 42 .

duced to a discrete mapping based on the nonlinear lattice Green's function introduced by Sievers and Takeno. ${ }^{21}$ The maximum residual error $\max _{m, n}\left|\phi_{m, n}\right|$ of the numerical solution $F_{m, n}$ of Eq. (8) never exceeded $10^{-10}$. 

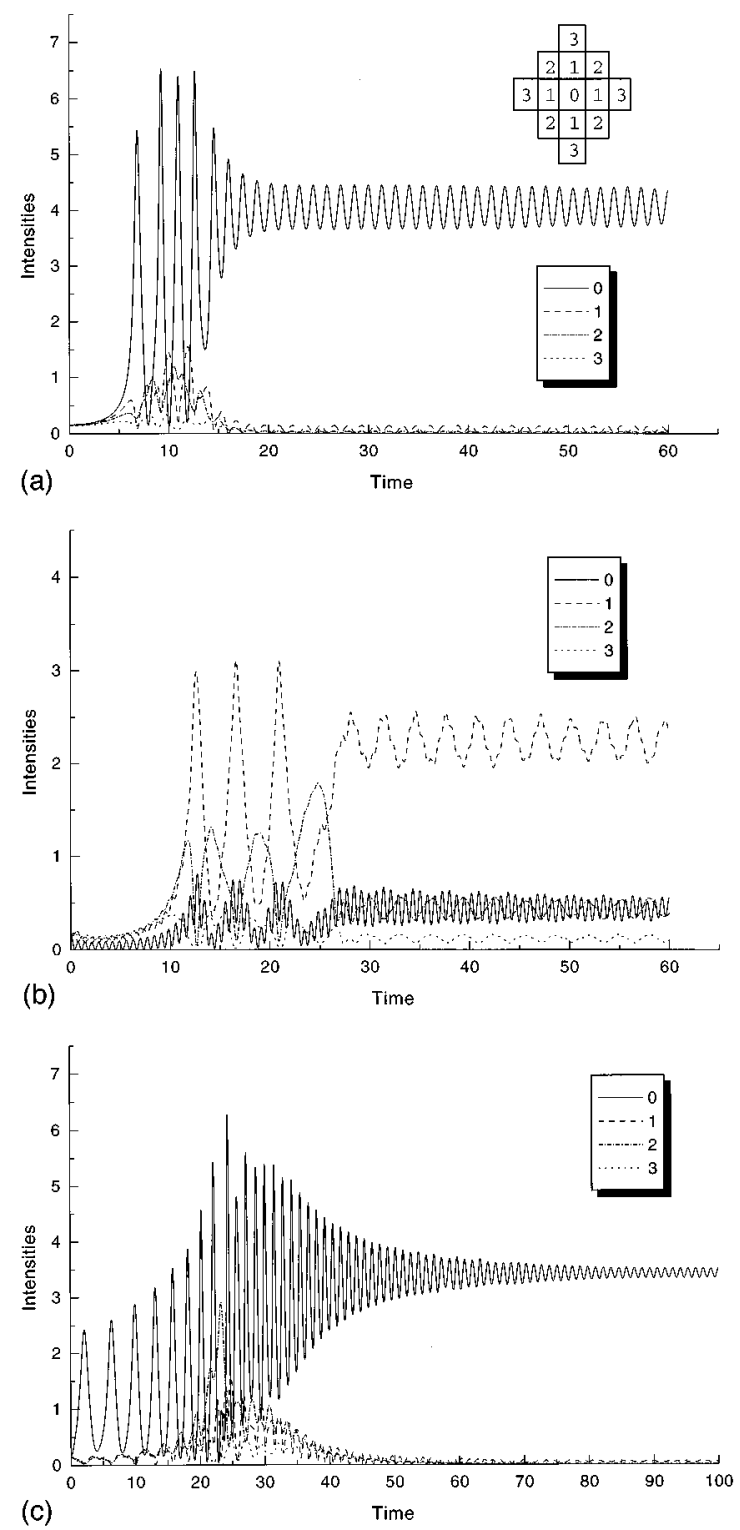

FIG. 6. Intensities versus time in the (a) homogeneous case $\omega_{0}=0$, (b) donor case $\omega_{0}=-4$, and (c) acceptor case $\omega_{0}=2$. Solid curve, central site (0); long-dashed curve site (1); dot-dashed curve, site (2); dashed curve, site (3). Sites numbers are defined in the inset.

Recently, the instability criterion for soliton solutions was proved for the pure DST case in Ref. 4 ( $\alpha=0$ in our notation). The solitons are unstable for $\partial N / \partial \omega<0$. The derivation of such a criterion was reported in Ref. 22 for the equations of the NSE type. For the pure DST case the same stability criterion for the one-dimensional discrete NSE with arbitrary power nonlinearity was obtained in Ref. 14. Here we extrapolate the stability criterion to the GDNSE, i.e., we assume that stable solitons correspond to the positive sign of the quantity $\partial N / \partial \omega$ or, which is the same, $\partial N / \partial \lambda$, where $\lambda=\omega^{1 / 2}$.

Figure 1(a) shows the excitation number $N$ for stationary solutions given by Eqs. (7) and (8) versus the spectral parameter $\lambda$ for a particular grid size: $32 \times 32$, for different values of $\alpha$. The value of the local maximum of $N$ increases with $\alpha$. On the other hand, the range in parameter $\lambda$ corre-
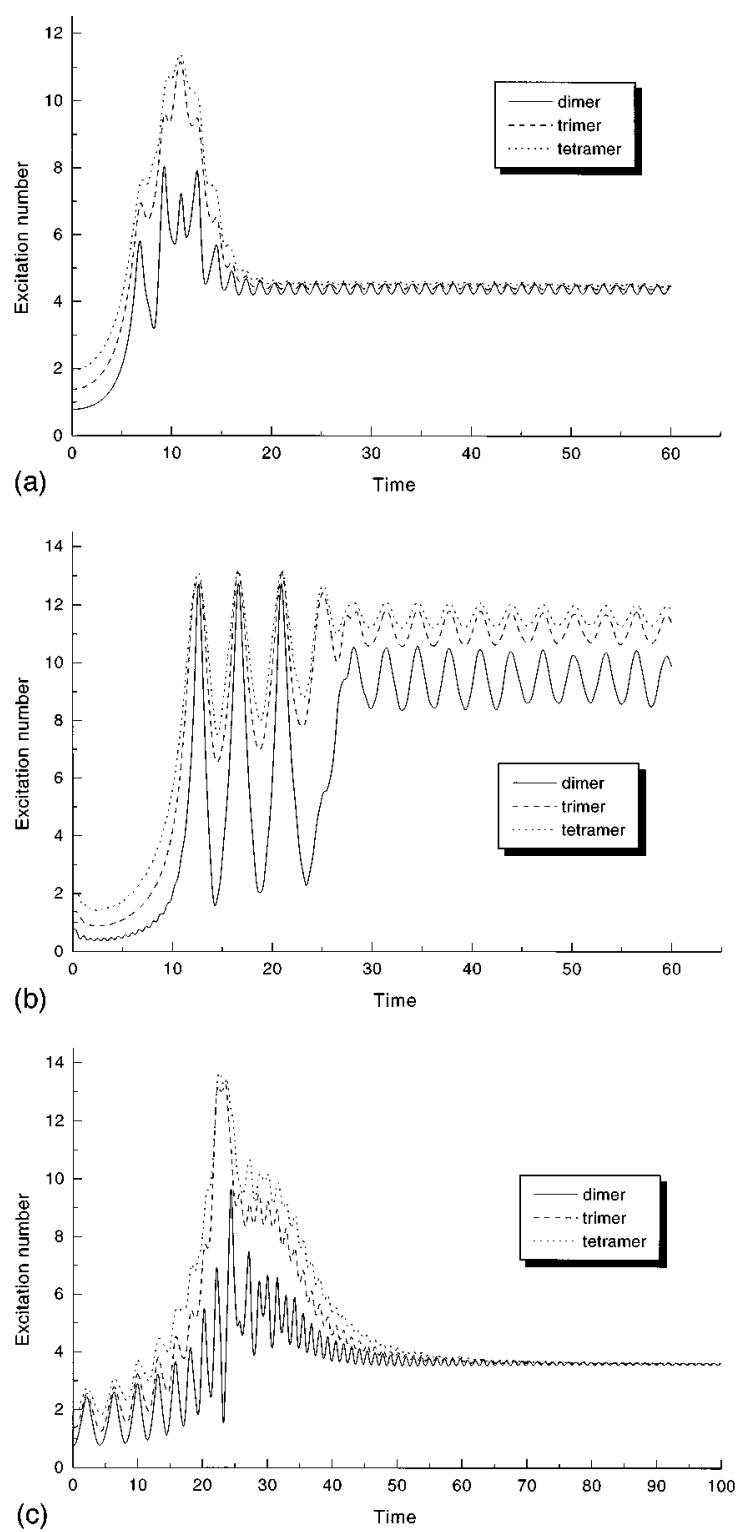

FIG. 7. Sum of intensities versus time in the (a) homogeneous case $\omega_{0}=0$, (b) donor case $\omega_{0}=-4$, and (c) acceptor case, $\omega_{0}=2$. Solid curve ( 5 sites, $\left.0+1\right)$; long-dashed curve $(9$ sites, $0+1$ $+2)$; short-dashed curve (13 sites, $0+1+2+3)$.

sponding to the negative slope of the curve $N(\lambda)$ narrows with the increase of $\alpha$. It means that in the case of AblowitzLadik solitons the instability region is smaller. In the limit of large $\lambda, \partial N / \partial \lambda>0$ for all $\alpha$; i.e., the stability property of the "narrow" soliton solutions is independent of $\alpha$.

Figure 1(b) illustrates the effect of a point defect on the stability of the soliton solutions in the DST cases. The soliton solutions were found for a lattice with a single acceptor introduced at the center of the lattice. The same curves, i.e., $N$ versus $\lambda$, are plotted for different acceptor frequency shifts. Note the stabilizing effect of the acceptor impurity. Apparently it can be explained by the attractive nature of the acceptor, i.e., the frequency shift in the acceptor case is positive $\left(\omega_{m, n}>0\right)$, therefore it acts physically as an attracting potential. For the donor case we have not found any localized stationary solutions with the maximum of intensity at the donor site. This is a consequence of the repelling nature of the donor defect. 


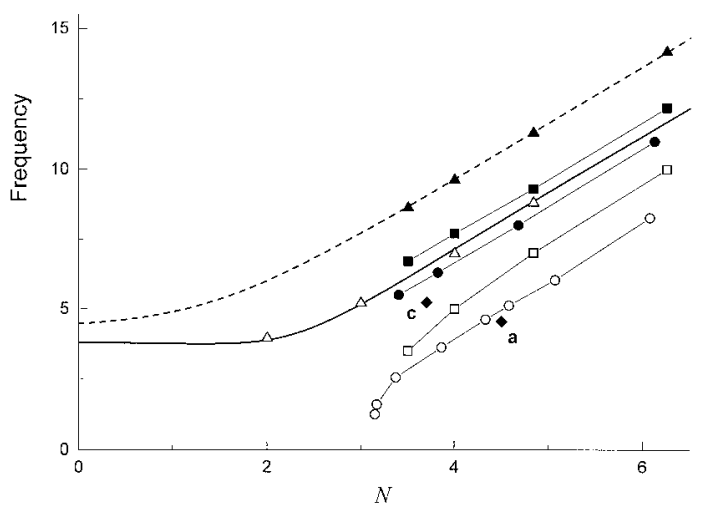

FIG. 8. Angular frequency $2 \pi / T$ versus the excitation number $N$ for the dimer system Eqs. (11) and (12); solid line, homogeneous case $\omega_{0}=0$; dashed line, acceptor case $\omega_{0}=2$. The triangles correspond to numerically integrate dimer truncation Eq. (2), squares correspond to trimer truncation, and circles correspond to frequencies of breather oscillations produced by the initially exciting only the central site in a large lattice $(64 \times 64)$. Open and full symbols show the homogeneous and the acceptor case, respectively. The diamonds labeled $a$ and $c$ show the frequencies of the post collapse oscillations in the homogeneous case [Figs. 6(a) and 7(a)] and the acceptor case [Figs. 6(c) and 7(c)].

In Fig. 2 the dependence of $N$ on the grid size is shown in the Ablowitz-Ladik case. In the infinite limit the influence of the boundary conditions becomes negligible and it is reflected in the narrowing of the dip in the region of small $\lambda$ with increasing number of grid points. The level of the flat plateau between the dip and the local maximum near $\lambda \approx 0.6$ approaches in a infinite lattice limit the asymptotic threshold value $N_{c}$ corresponding to the continuum groundstate soliton. Note that in this case there is a finite interval near the threshold value in which three values of the spectral parameter $\omega$ give the same value of $N$. We conjecture that only the smaller and the larger values of $\omega$ (for which $\partial N / \partial \omega>0)$ correspond to stable solutions, i.e., bistability occurs. We have obtained numerical evidence for that conjecture using the soliton solution as an initial condition in simulations of the full dynamical equations (2).

For our further consideration the stability of the narrow solitons $(\omega>1)$ corresponding to the right branch of the curve $N(\lambda)$ with the positive slope, is of primary importance. These stable states will appear as the final states of the quasicollapse evolution of broad initial conditions.

\section{QUASICOLLAPSE OF THE LOCALIZED EXCITATIONS}

The localization (quasicollapse) and pinning of the traveling waves are the important questions in applications. The basic model Eq. (2) possesses in the absence of defects traveling-wave solutions in the form of monochromatic waves

$$
\begin{gathered}
U_{m, n}=A \exp \left(i \Omega t+i m \kappa_{m}+i n \kappa_{n}\right) \\
\Omega=4\left(\sin ^{2} \kappa_{m}+\sin ^{2} \kappa_{n}\right)+2\left[1-\alpha+\frac{\alpha}{2}\left(\cos \kappa_{m}+\cos \kappa_{n}\right)\right]|A|^{2} .
\end{gathered}
$$

It implies that broad smooth pulses modulated in space will propagate, at least initially, similarly to the monochromatic wave Eqs (9). In this section we describe the results of the numerical simulations of the localization dynamics of the initially standing (Sec. IV A) and moving (Sec. IV B) excitations. We study the evolution of a localized pulse given initially as

$$
\begin{gathered}
U(m, n, t=0)=A(m, n) \exp \left[i \kappa_{m}(m-\bar{m})+i \kappa_{n}(n-\bar{n})\right], \\
A(m, n)=A_{0} \exp \left[-\left(\frac{m-\bar{m}}{m_{0}}\right)^{2}-\left(\frac{n-\bar{n}}{n_{0}}\right)^{2}\right] .
\end{gathered}
$$

In order to investigate the dynamical evolution of the initial conditions Eqs. (10) we solved Eq. (2), using a standard fourth-order Runge-Kutta scheme. To check the accuracy we calculate the relative errors $\epsilon_{N}=2|N(t)-N(0)| / N(0)$ and $\epsilon_{H}=2|H(t)-H(0)| / H(0)$. We have kept $\epsilon_{N}$ and $\epsilon_{H}$ less that $10^{-9}$ and $10^{-5}$, respectively. In most of our runs we have used a lattice of the size $65 \times 65$ with the indices $m, n$ varying within the range $[-M / 2, M / 2]$ with $M=64$. Zero boundary conditions are implied. Since the primary goal of the simulations is a study of the contraction dynamics, i.e., the events of interest are very localized (usually within a box of the width of a few lattice sites), the influence of boundaries is typically limited to the reflection of the weak radiation going out from the collapsing pulse. A lattice two times larger with $M=128$ and the same initial conditions is simulated to verify the absence of significant boundary effects in suspicious cases.

Contrary to the continuum limit Eq. (6), the discrete model demonstrates a much richer variety of localization dynamics. It is worth recalling that in the case of continuum $2 \mathrm{D}$ NSE's there are only two alternatives that can be realized; either collapse in finite time or dispersive spreading of the initial pulse all over space.

In the following discussion of the results we restrict ourselves to the consideration of the DST case. However, similar calculations were carried out for the AL case. We discuss briefly the qualitative differences at the end of the present section.

\section{A. Standing excitations}

We first investigate the development of quasicollapse of standing excitations in the presence of the point defects; thus we start with the initial condition Eq. (10) with $\kappa_{m}=\kappa_{n}=0$. In all cases, we have employed symmetric pulses $\left(m_{0}=n_{0}\right)$ and a symmetric lattice. The quasicollapse on a homogeneous lattice was investigated in details in Refs. 3 and 23. In the present subsection we emphasize the influence of point defects on the quasicollapse and post-collapse evolution.

Figure 3 shows the typical dynamics of the standing solution in the homogeneous DST case $(\alpha=0$, no defects introduced). We observe that the initially circular symmetric excitation develops into a breatherlike structure oscillating between the center of the initial excitation and the few nearest-neighbor sites (see also Refs. 3 and 4). Adding a defect at the center of the initial excitation, we obtain the evolution shown in Figs. 4 and 5 for the donor and acceptor cases, respectively. In the donor case (Fig. 4) four neighbor- 
Time $=0$
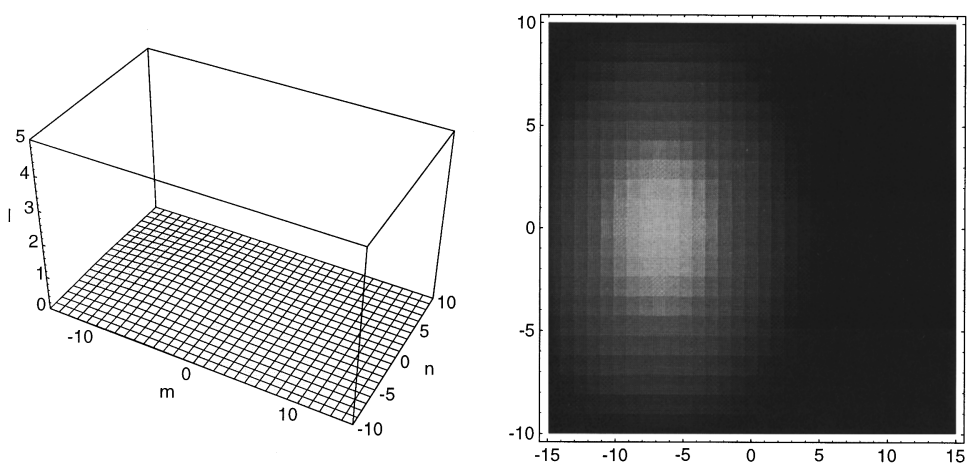

Time $=18$
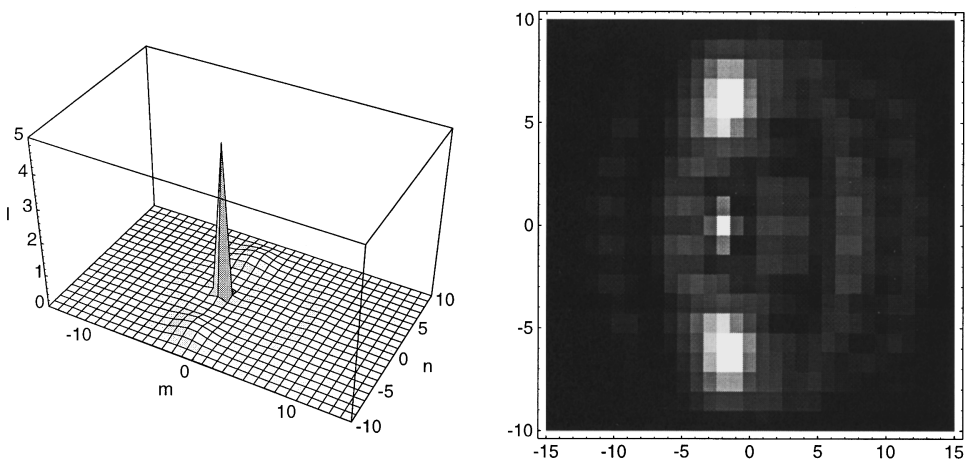

Time $=24$
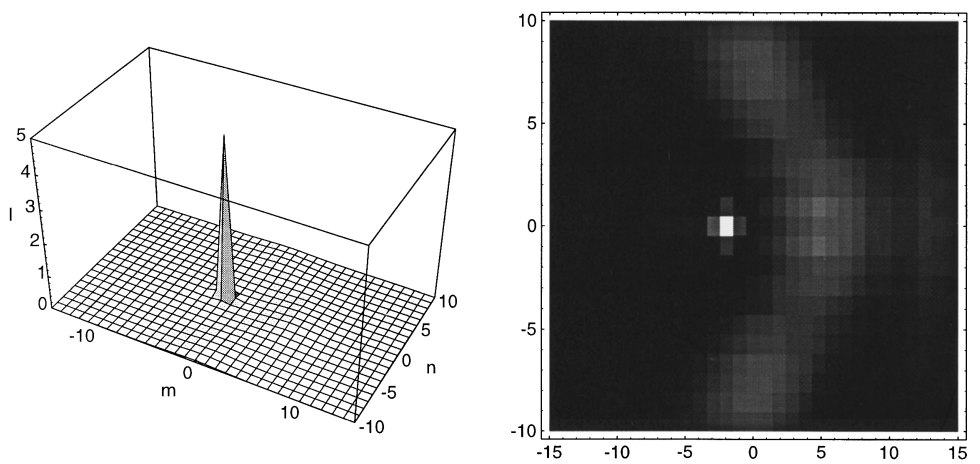

FIG. 9. Dynamics of a moving solution in the homogeneous DST case Eq. (2) with $\omega_{0}=0$, $\alpha=0$. Initial condition, Eq. (10), with $A_{0}=0.4, m_{0}=n_{0}=6, \kappa_{n}=0, \kappa_{m}=0.1 \pi$, and $(\bar{m}, \bar{n})=(-7,0) ; t=0,18$, and 24 . ing spikes are excited without a significant central spike. As a result of the repulsion from the donor site the creation of four spikes turns out to be sensitive to the symmetry of the system, e.g., the relative position of the donor site, symmetry of the lattice, or symmetry of the initial distribution. In the asymmetrical situation two spikes may appear instead of four. In the acceptor case (Fig. 5) a central spike develops. In contrast to the defect less case (Fig. 3), a broad pedestal background excitation develops due to the attraction by the acceptor. In this case the evolution is insensitive to asymmetries.

To illustrate the fine structure of the central region of the contracted excitation, we plot the time dependence of the intensities $I_{m n}=\left|U_{m n}\right|^{2}$ for several central cites. In Fig. 6 we have plotted the time evolution of the sites in the neighborhood of the central site in the homogeneous, donor, and acceptor cases. Similarly, Fig. 7 illustrates the sums of the intensities of the neighboring sites. We observe that in the donor case the dominant part of the intensity is contained in the nearest neighbors (site 1) [Fig. 6(b)]. In the homogeneous case and the acceptor case the central site will contain the main portion of the intensity [Figs. 6(a) and 6(c)]. Furthermore, the intensity oscillations are seen to persist, indicating the breather nature of the excitations. In view of the observed behavior we may expect that the dynamics of the collapsed state is simply governed by a dimer approximation as was also suggested in Ref. 4. It is immediately seen that only in the homogeneous case and the acceptor case may the dimer truncation approximate the results of the numerical simulations. This is due to the fact that only in those cases the total intensity at the central site and the nearest neighbors $(0+1)$ is approximately constant. A simple analysis of the observed oscillations can thus be performed by truncating the system such that only the five central sites ( $U_{0,0}, U_{ \pm 1,0}$, and $U_{0, \pm 1}$ ) are considered (see also Ref. 4). Assuming additionally that the excitation is completely symmetric $\left(U_{ \pm 1,0}=U_{0, \pm 1}=\frac{1}{2} \phi_{1}\right.$ and $\left.U_{0,0}=\phi_{0}\right)$ we get the system of equations

$$
i \dot{\phi}_{0}+2 \phi_{1}-4 \phi_{0}+2\left|\phi_{0}\right|^{2} \phi_{0}+\omega_{0} \phi_{0}=0,
$$


Time $=0$
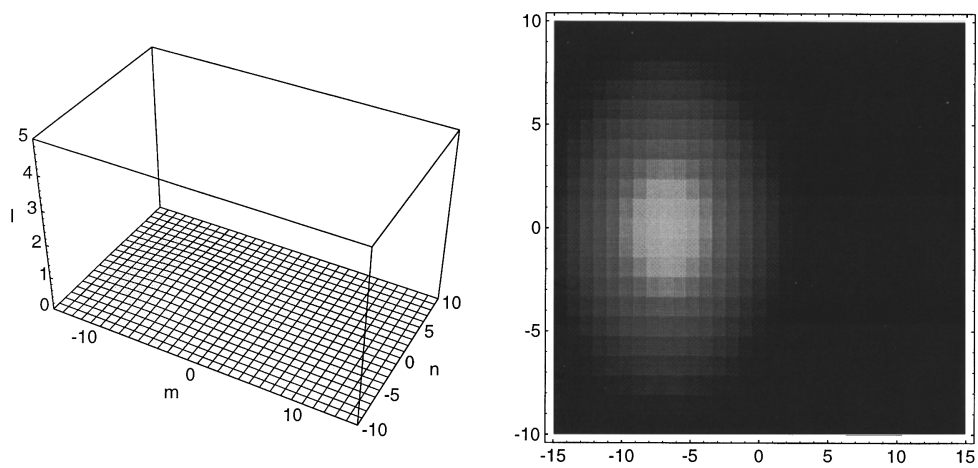

Time $=12$
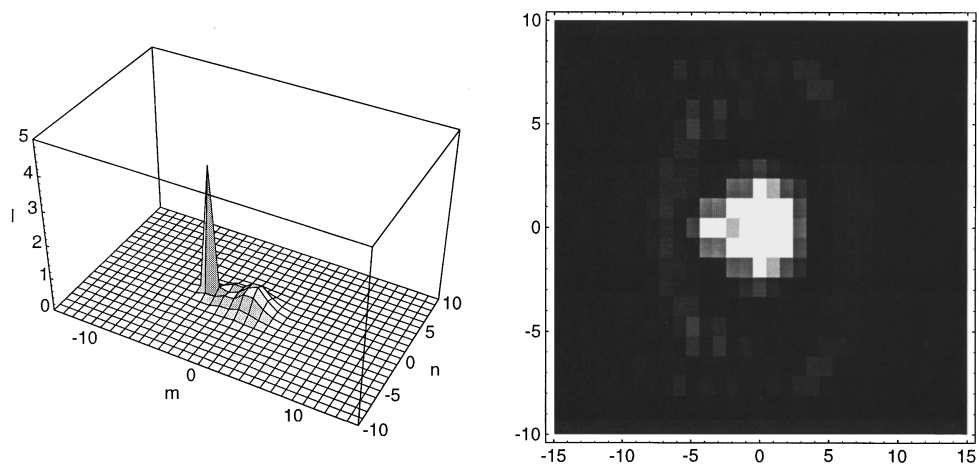

Time $=48$
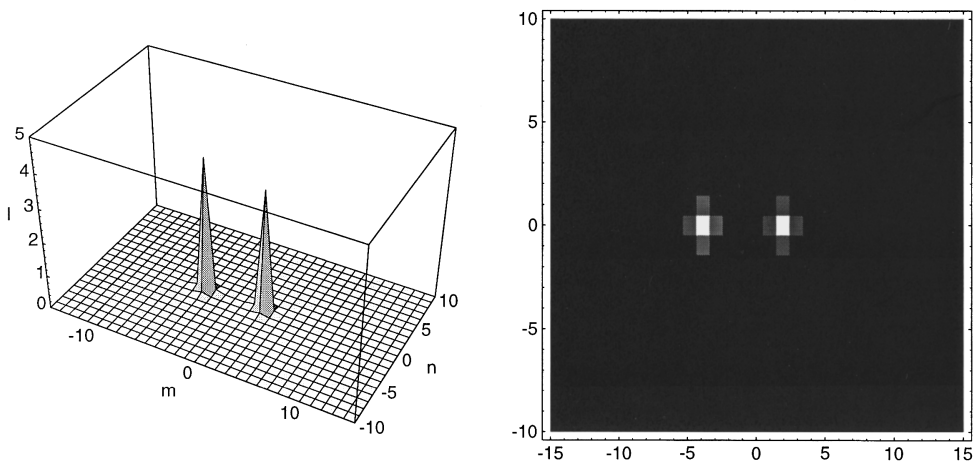

FIG. 10. Dynamics of a moving solution in homogeneous DST case Eq. (2) with $\omega_{0}=0$, $\alpha=0$. Initial condition, Eq. (10), with $A_{0}=0.4, \quad m_{0}=n_{0}=8, \quad \kappa_{n}=0, \quad \kappa_{m}=0.1 \pi, \quad$ and $(\bar{m}, \bar{n})=(-7.0) ; t=0,12$, and 48 .

$$
i \dot{\phi}_{1}+2 \phi_{0}-4 \phi_{1}+\frac{1}{2}\left|\phi_{1}\right|^{2} \phi_{1}=0 \text {. }
$$

These equations are easily analyzed introducing the four real variables (Bloch variables)

$$
\begin{gathered}
x=\phi_{0} \phi_{1}^{*}+\phi_{1} \phi_{0}^{*}, \quad y=i\left(\phi_{0} \phi_{1}^{*}-\phi_{1} \phi_{0}^{*}\right), \\
z=\left|\phi_{0}\right|^{2}-\left|\phi_{1}\right|^{2}, \quad N_{d}^{2}=\left(\left|\phi_{0}\right|^{2}+\left|\phi_{1}\right|^{2}\right)^{2}=x^{2}+y^{2}+z^{2},
\end{gathered}
$$

where $N_{d}$ is an integral of motion. From here we get the equation for $z$

$$
\ddot{z}=\delta E-\left(\delta^{2}+16-\frac{5}{4} E\right) z-\frac{15}{8} \delta z^{2}-\frac{25}{32} z^{3},
$$

where $\delta=\omega_{0}+\frac{3}{4} N_{d}$ and $E$ is a constant determined by the initial values $E=\delta z(0)+4 x(0)+\frac{5}{8} z(0)^{2}$. From the solution of Eq. (14) one can express the period $T$ of the dimer oscillations in terms of the complete elliptic integral of the first kind. Figure 8 shows the angular frequency $2 \pi / T$ versus excitation number $N$ for both the homogeneous case (solid line) and the acceptor case (dashed line). However, a direct comparison between the frequencies of the dimer oscillation and the breatherlike oscillations in the post-collapse stage as in Fig. 6 shows the full lattice simulations give frequencies significantly below the values predicted by the dimer model for both the homogeneous case and the acceptor case (shown by diamonds with labels $a$ and $c$, respectively, in Fig. 8).

To clarify this discrepancy we have numerically solved Eq. (2) truncated to a dimer [also Eqs. (11) and (12)] with the initial excitation on only the central site. This develops a breatherlike solution with the main frequency, obtained from the first peak in the frequency spectrum, that agrees with the one predicted from the solution of Eq. (14). These frequencies are shown by open triangles (homogeneous case) and full triangles (acceptor case) in Fig. 8. Similarly we have obtained the main frequencies for the breatherlike oscillations in a system truncated to a symmetrical "trimer'" (the 


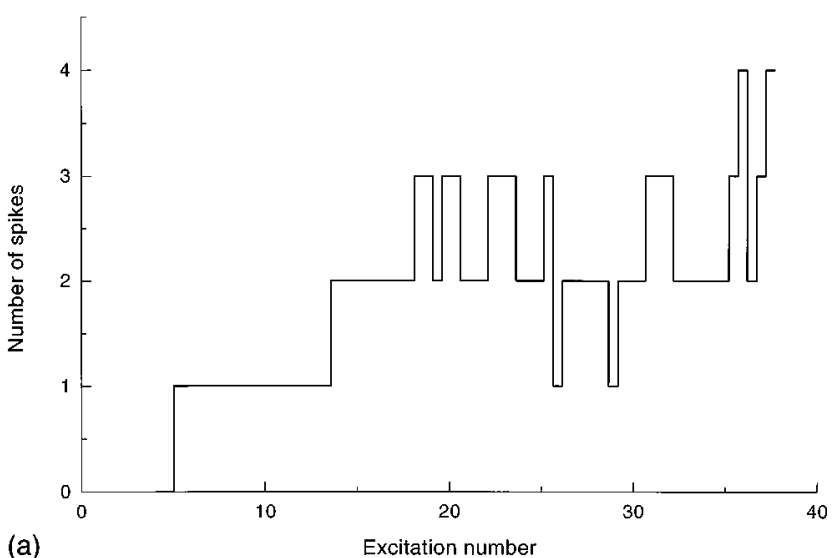

(a)

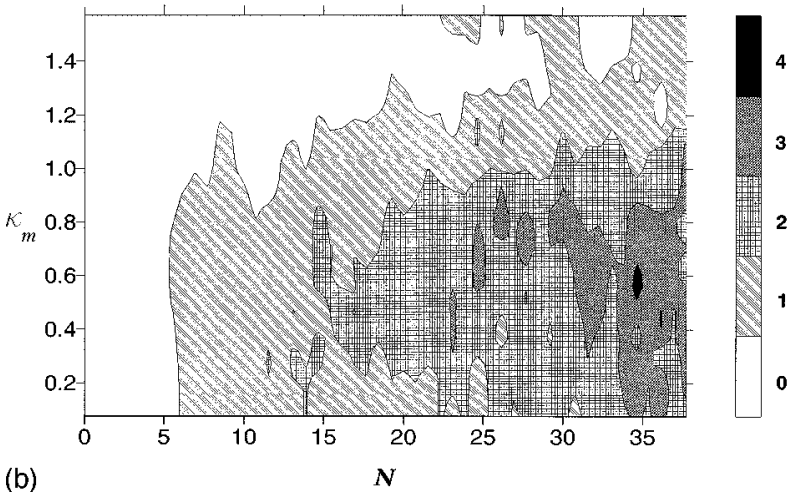

(b)

FIG. 11. Number of spikes $\nu$ created during the quasicollapse of the moving initial pulse. Initial condition, Eq. (10), with $m_{0}=n_{0}=8$ and $(\bar{m}, \bar{n})=(-7,0)$. (a) Dependence of $\nu$ on the excitation number $N$ for fixed initial modulation $\kappa_{n}=0, \kappa_{m}=0.1 \pi$. (b) Contours of $\nu$ as a function of $N$ and $\kappa_{m}$.

sites 0,1 , and 2 in the inset on Fig. 6). These frequencies, shown by open squares for homogeneous case and full squares for acceptor case, are observed to be between the dimer frequency and the frequency for post-collapse breather. The more sites we include in the truncation the closer we get to the frequency of the post-collapse breather, which indeed is found to agree with frequencies shown by circles in Fig. 8 that develop in the full system Eq. (2), when initially only a central site is excited as described in Ref. 4. The same conclusion applies for the acceptor case, where the frequency for the breather developing from the central excitation is shown by full circles in Fig. 8. We may thus conclude that the collapse of the initially broad pulse leads to a breatherlike solution with the main intensity in one central site, which behaves like the breather developing from excitation of the central site alone. However, the detailed dynamics cannot be described by a simple dimer or even trimer approximation. The arguments above apply only to the homogeneous case and the case with an acceptor defect; for a donor defect the dynamics is even more complicated due to its repulsive nature as discussed in connection with Figs. 6 and 7.

\section{B. Moving excitations}

The evolution of moving excitations is investigated by using the initial condition Eq. (10) with $\kappa_{m} \neq 0$ and $\kappa_{n}=0$, i.e., excitations are moving in the " $m$ direction.", The evo- lution is strongly dependent on the initial excitation number $N$ and modulation vector $\kappa_{m}$. Also, here we start with a broad initial distribution. In general, we observe that the pulse contracts as it moves along. When a spike of width of few sites is formed it slows down and get pinned at a particular site. The remaining part of the pulse continues to move and contract and may subsequently leave another pinned spike. This scenario continues until the main part of the initial pulse is pinned at different sites, and when the rest of the pulse is unable to contract it is dispersing.

Figures 9 and 10 show the evolution of moving excitations in the homogeneous DST case for different excitation numbers $N \simeq 9$ and 16 , respectively. In the first case one spike is dropped after six sites at $(0,-1)$. In the latter case two spikes are dropped after three and nine sites with slightly decreasing amplitudes. We note that the excitation number in Fig. 9 is smaller that twice the threshold value $N_{c}=5.86$, thus only permitting the formation of a single spike, while the excitation number in Fig. 10, being smaller than three times the threshold value, permits formation of two spikes. (A simulation of a moving solution with the same amplitude and modulation wave vector $n_{0}=m_{0}=10$ corresponding to excitation number $N \simeq 25$ showed the creation of three spikes.) We observe that the number of spikes $\nu$, created during the contraction of the initial pulse increases with the excitation number for a fixed value of the modulation wave vector $\kappa_{m}$. The dependence of $\nu$ on the excitation number for $\kappa_{m}=0.1 \pi$ is plotted in Fig. 11(a). While the general trend is an increase in the number of the created spikes with increasing $N$, the fine structure of the curve is quite complicated. The sharp resonant peaks occur on the broader background pedestal at particular values of the excitation numbers. We explain these resonances by a certain relation between the oscillation period of the created breather during the collapse and the transition time of the smooth broad background moving through the collapsing site. In Fig. 11(b) we show the contour of $\nu$ as a function of excitation number and modulation wave vector: $\nu(N,|\kappa|)$. Note from Fig. 11 that the critical value $N_{c}$ appears to be a threshold value for the creation of a single spike in the limit of small modulation wave vectors. It seems natural because the initial pulse is taken to be smooth; therefore the initial dynamics of the contraction of the moving pulse is close to the continuum limit. Thus this scenario defines the minimum threshold value of the excitation number that is necessary to create the first spike. On the other hand, the critical value $N_{c}$ gives an estimate from above for the excitation number trapped in the pinned spike. Our results show that the excitation number trapped in the single standing spike is always less than $N_{c}$; usually it is around 4.

The pinned excitation created in the post-collapse stage of the moving pulse only have very weak oscillations of the central site intensity in contrast to the post-collapse evolution of the standing excitation. They are not breatherlike, but resemble the stationary stable narrow solitonlike excitations discussed in Sec. III. The qualitative difference between these two cases lies in the velocity of the weak background surrounding the pinned spike. In the moving cases a weak pedestal keeps moving out of the pinning site, while in the case of standing quasicollapse there is permanent strong in- 
Time $=0$
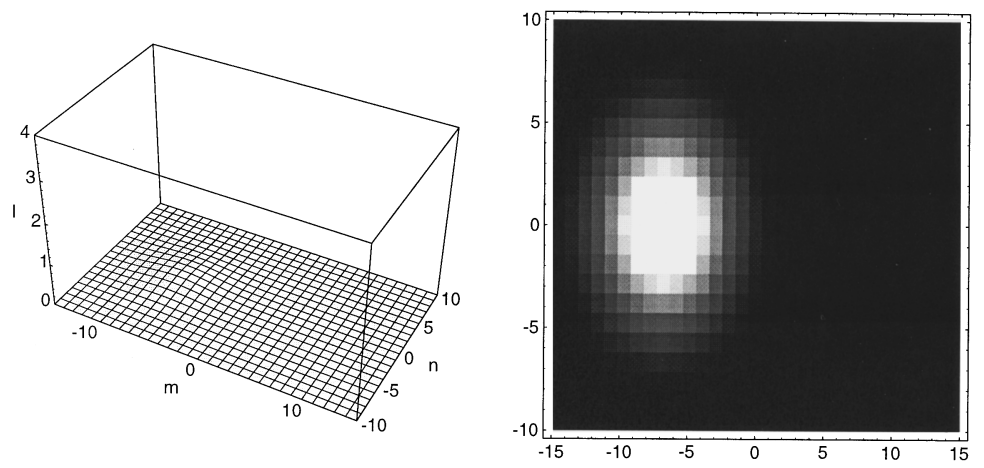

Time $=6$
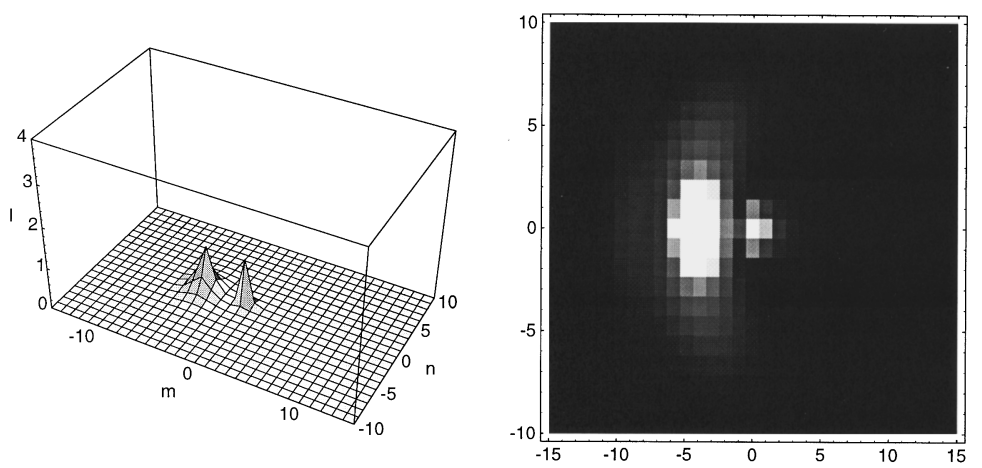

Time $=24$
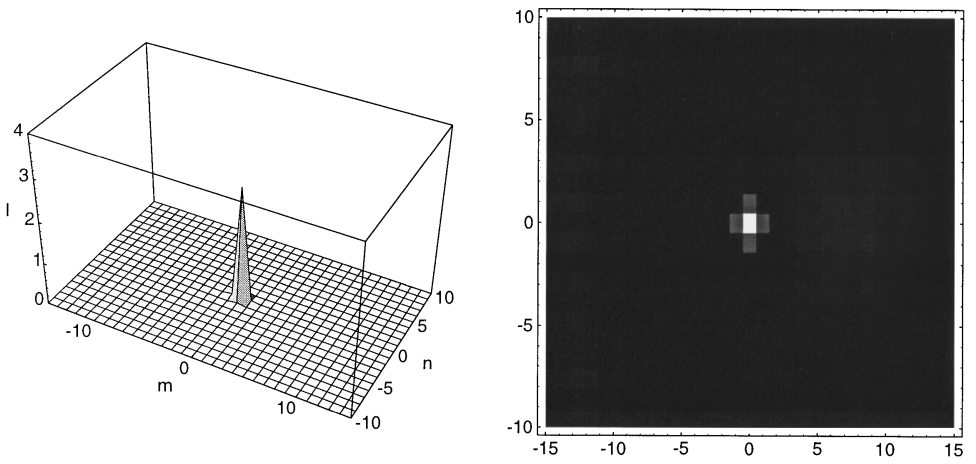

FIG. 12. Dynamics of a moving solution in the inhomogeneous acceptor DST case Eq. (2) with $\omega_{0}=2, \alpha=0$. Initial condition, Eq. (10), with $A_{0}=0.4, m_{0}=n_{0}=6, \kappa_{n}=0, \kappa_{m}=0.1 \pi$, and $(\bar{m}, \bar{n})=(-7,0) ; t=0,6$, and 24. Defect at $(0,0)$. teraction between the central site and its pedestal, which eventually leads to creation of a breatherlike structure.

Introducing defects into the DST lattice, we obtain the results for the dynamics of the moving solution shown in Figs. 12-14. In Fig. 12 head-on collision with an acceptor leads to the creation of a bound state at the site of the defect. For high initial excitation numbers we have observed the formation of pinned spikes beyond the acceptor. In general, the threshold for forming more spikes is higher for the acceptor case than for the homogeneous case. The influence of acceptor on the collapse dynamics is, however, depending on different parameters such as acceptor strength and position and lattice symmetry and, in addition, on the pulse parameters, in a very complicated manner and a detailed study is beyond the scope of this paper.

Figure 13 shows a similar collision with a donor defect. In this case a bound state is created next to the donor [i.e., at $(m, n)=(0,-4)]$. This difference of behavior in the acceptor and the donor case is caused by the reflection from the strong donor defect. In the acceptor case initially no reflection occurs. Figure 14 shows the collision with a donor for an excitation with $N \simeq 6.2$, which is slightly above the threshold value. In this case the excitation is seen to disperse due to the repulsion of the donor. Note that in the absence of the defect the same initial condition collapses.

Investigations of collapse dynamics similar to the ones described above were also carried out for the AL case [Eq. (2) with $\alpha=1]$. For both the standing and moving excitations we found qualitatively the same behavior. However, the intensity of the pinned spikes is considerably higher, which is due to the fact that the excitation number, which is still limited by $N_{c}$, for the $\mathrm{AL}$ case reads $N \simeq 2 \ln (1$ $+\frac{1}{2}\left|U_{0,0}\right|^{2}$ ) with almost all intensity trapped at one site. From here one can estimate the central site intensity as

$$
\left|U_{0,0}\right|^{2}=2 e^{N / 2}-2 \approx 16
$$

for $N=N_{c} \approx 5.86$. A detailed comparison of the quasicollapse dynamics in DST and AL cases is presented in Ref. 23. 
Time $=0$
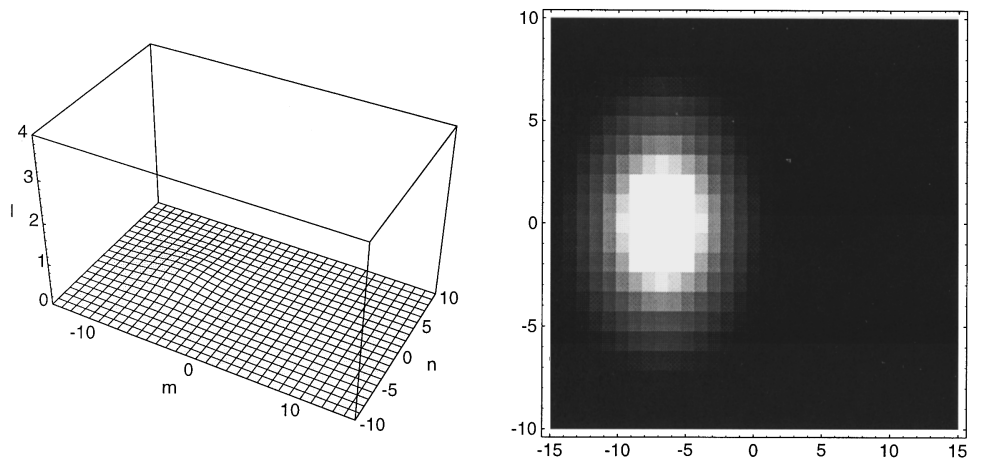

Time $=18$
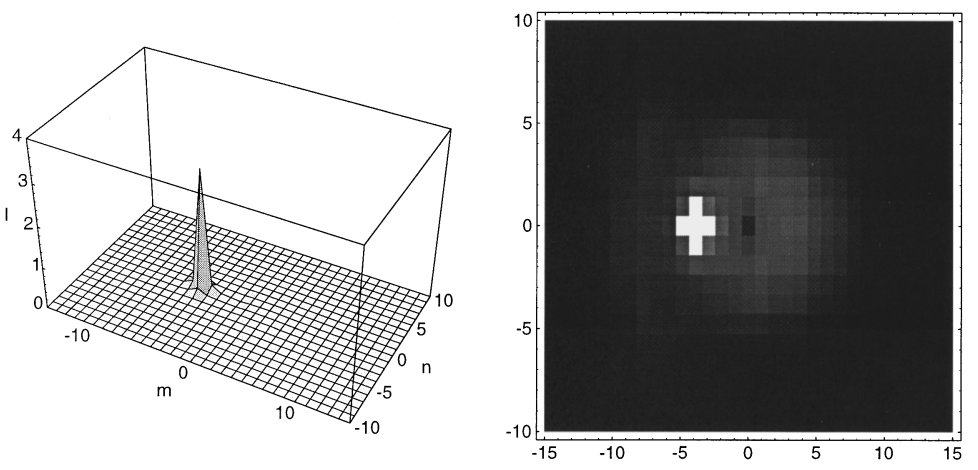

Time $=24$
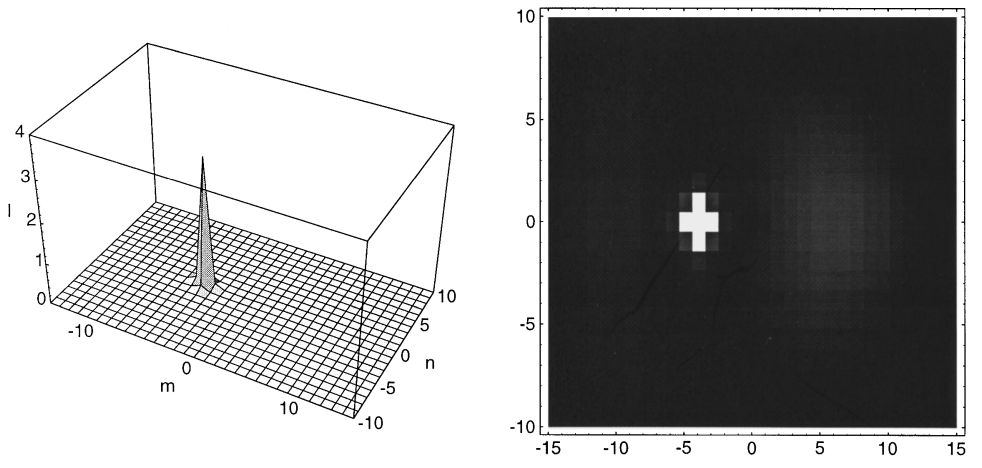

Time $=48$
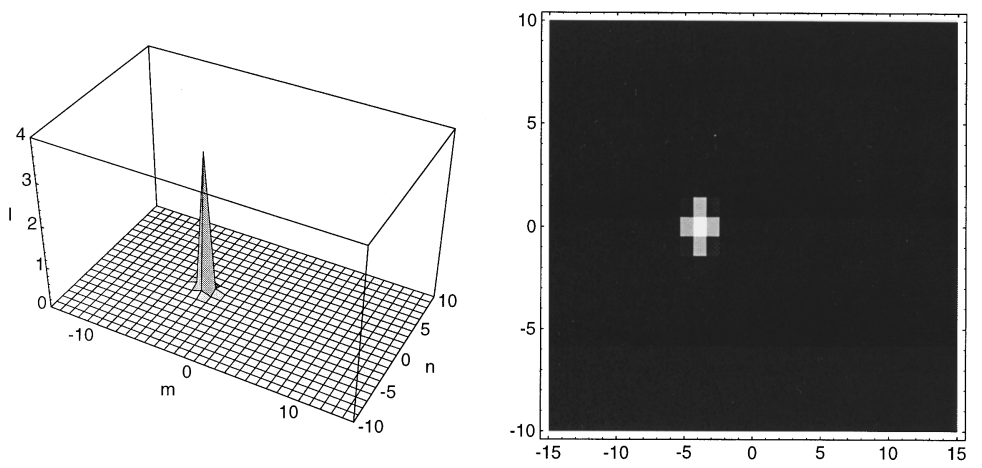

FIG. 13. Dynamics of a moving solution in the inhomogeneous donor DST case Eq. (2) with $\omega_{0}=-2, \alpha=0$. Initial condition, Eq. (10), with $A_{0}=0.4, m_{0}=n_{0}=6, \kappa_{n}=0, \kappa_{m}=0.1 \pi$, and $(\bar{m}, \bar{n})=(-7,0) ; t=0,18,24$, and 48. Defect at $(0,0)$.

\section{SUMMARY AND CONCLUSIONS}

We have studied the nonlinear dynamics of intense envelope pulses within the context of a generalized discrete nonlinear Schrödinger equation incorporating the on-site nonlinearity, the linear and nonlinear coupling between nearest neighbors, and the presence of point defects. We have found the generalized two-dimensional discrete solitons and have shown their significant role in the final stage of the quasicollapse dynamics. The influence of the nonlinear coupling terms (AL term) on the stability of soliton solutions is to increase the stability regime. Also, an acceptor point defect has a stabilizing effect on soliton solutions. The dynamics of 
Time $=0$
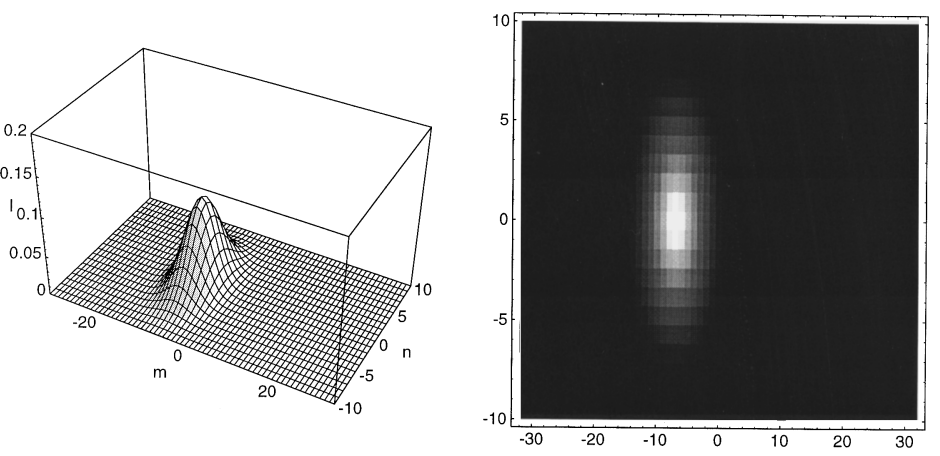

Time $=18$
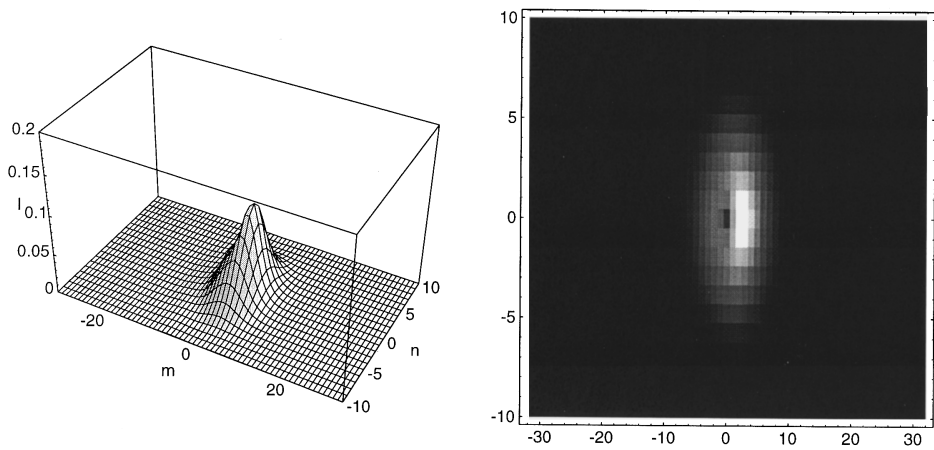

Time $=24$
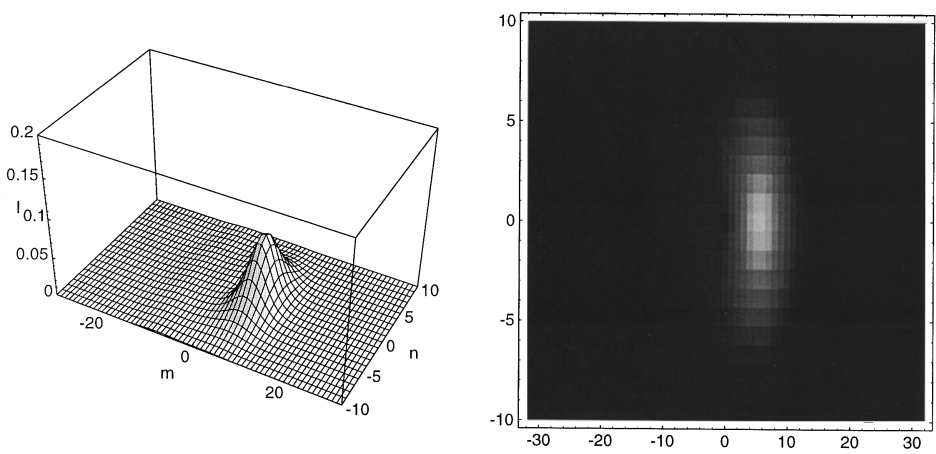

FIG. 14. Dynamics of a moving solution in the inhomogeneous donor DST case Eq. (2) with $\omega_{0}=-2$ at $(m, n)=(-7,0), \alpha=0$. Initial condition, Eq. (10), with $A_{0}=0.33, m_{0}=n_{0}=6, \kappa_{n}$ $=0, \kappa_{m}=0.1 \pi$, and $(\bar{m}, \bar{n})=(-7,0) ; t$ $=0,18,24$, and 48. Defect at $(0$,$) .$

Time $=48$
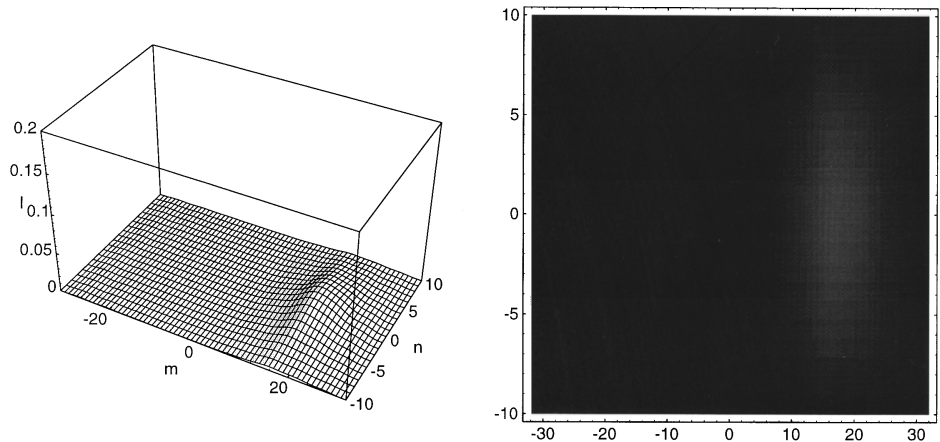

contracting moving pulses is investigated in detail. We have found the conditions of quasicollapse and investigated the transition from the initial states that are close to the continuum limit to the superposition of essentially discrete immobile narrow states. The characteristic feature of the discrete quasicollapse of a moving pulse is the splitting of the initially moving broad pulse into a track of the standing narrow structures that have nearly fixed trapped energy. This phenomenon defines the finite lifetime of the initial pulse and its finite propagation-penetration length. The significant part of the initial energy of the pulse in the range of 30-60\% of the initial value is trapped at certain sites, while the rest is spread dispersively over the whole lattice. The influence of point defects in the form of on-site linear frequency shifts on the quasicollapse dynamics has also been studied. A very complicated behavior depending in detail on several param- 
eters was observed. But, in general, the acceptor-type defect tends to promote collapse and attract the excitation to the defect site, while the donor defect due to its repulsive nature may counteract the collapse and repel the excitation.

We have mainly concentrated our investigations on the DST case, which has more direct applications than the AL version of the GDNSE, as will be apparent from the discussion in the Introduction. Our results have obvious consequences for the understanding of the energy transport and localization in nonlinear 2D lattices.

The 2D DST lattice has, for instance, been proposed as a model of monolayer Scheibe aggregates by several authors (see Refs. 24, 17, and 18 for a survey). These molecular aggregates have a highly ordered and compact structure and are produced by Langmuir-Blodgett techniques. In cyanine aggregates Möbius and Kuhn ${ }^{25}$ have observed highly efficient energy transfer over large distances between donor and acceptor molecules, the former and the latter being oxacyanine and thiacyanine, respectively. The energy transfer is assumed to be carried out by coherent excitons moving on the

*Permanent address: Institute of Theoretical Physics, Metrologicheskaya 14 B, 252143 Kiev, Ukraine.

†Permanent address: Institute for Automation and Electrometry, 630090 Novosibirsk, Russia.

${ }^{1}$ Nonlinear Coherent Structures in Physics and Biology, edited by K.-H. Spatchek and F. G. Mertens (Plenum, New York, 1994); Future Directions of Nonlinear Dynamics in Physics and Biology, edited by P. L. Christiansen, J. C. Eilbeck, and R. D. Parmentier (Plenum, New York, 1993).

${ }^{2}$ R. S. MacKay and S. Aubry, Nonlinearity 7, 1623 (1994).

${ }^{3}$ V. K. Mezentsev, S. L. Musher, I. V. Ryzhenkova, and S. K. Turitsyn, Pis'ma Zh. Éksp. Teor. Fiz. 60, 815 (1994) [JETP Lett. 60, 829 (1994)].

${ }^{4}$ E. W. Laedke, K.-H. Spatchek, V. K. Mezentsev, S. L. Musher, I. V. Ryzhenkova, and S. K. Turitsyn, Pis'ma Zh. Éksp. Teor. Fiz. 62, 652 (1995) [JETP Lett. 62, 677 (1995)].

${ }^{5}$ M. Salerno, Phys. Rev. A 46, 6856 (1992).

${ }^{6}$ D. Cai, A. R. Bishop, and N. Grønbech-Jensen, Phys. Rev. Lett. 72, 591 (1994).

${ }^{7}$ J. C. Eilbeck, P. S. Lomdahl, and A. C. Scott, Physica D 16, 318 (1985).

${ }^{8}$ M. J. Ablowitz and J. F. Ladik, J. Math. Phys. 17, 1011 (1976); Stud. Appl. Math. 55, 213 (1976).

${ }^{9}$ A. Scott, Phys. Rep. 217, 1 (1992).

${ }^{10}$ A. Aceves, G. Luther, C. De Angelis, A. M. Rubenchik, and S. K. Turitsyn, Phys. Rev. Lett. 75, 73 (1995); Physica D 87, 262 (1995)

${ }^{11}$ D. Hennig, N. G. Sun, H. Gabriel, and G. P. Tsironis, Phys. Rev. E 52, 255 (1995). aggregate until they become absorbed by the doped acceptor molecules. The efficiency of this transfer mechanism increases with temperature. In the nonlinear model of the Scheibe aggregate the Möbius-Kuhn coherent exciton is represented by the moving ground-state solution. In a sequel to the present paper we plan to study the influence of thermal fluctuations on the mobility of the moving ground-state solutions. Furthermore, the results obtained in the present paper are directly applicable to the carrier wave propagation dynamics in two-dimensional fiber arrays. ${ }^{10}$

\section{ACKNOWLEDGMENTS}

We acknowledge the support by INTAS Grant No. 93139 and the Danish Natural Research Council SNF (Grant No. 11-0921-1). V.K.M. and Y.B.G. wish to thank their colleagues at the Department of Optics and Fluid Dynamics at the Ris $\phi$ National Laboratory and at the Institute for Mathematical Modelling at the Danish Technical University for their kind hospitality.

${ }^{12}$ R. Scharf and A. R. Bishop, Phys. Rev. A 43, 6535 (1991).

${ }^{13}$ P. Marquie, J. M. Bilbault, and M. Remoissenet, Phys. Rev. E 51, 6127 (1995).

${ }^{14}$ E. W. Laedke, K.-H. Spatchek, and S. K. Turitsyn, Phys. Rev. Lett. 73, 1055 (1994).

${ }^{15}$ O. Bang, J. Juul Rasmussen, and P. L. Christiansen, Nonlinearity 7, 205 (1994); Physica D 68, 169 (1993).

${ }^{16}$ X. D. Cao and B. A. Malomed, Phys. Lett. A 206, 177 (1995).

${ }^{17}$ Yu. B. Gaididei, K. Ф. Rasmussen, and P. L. Christiansen, Phys. Rev. E 52, 2951 (1995).

${ }^{18}$ Yu. B. Gaididei, K. Ф. Rasmussen, and P. L. Christiansen, Phys. Lett. A 203, 175 (1995).

${ }^{19}$ J. Juul Rasmussen and K. Rypdal, Phys. Scr. 33, 481 (1986).

${ }^{20}$ V. I. Petviashvili and O. A. Pokhotelov, Solitary Waves in Plasma and in the Atmosphere (Energoatomizdat, Moscow, 1992); L. A. Abramyan, Yu. A. Stepanyants, and V. I. Shrira, Dokl. Akad. Nauk USSR 327, 460 (1992) [Sov. Phys. Dokl. 37, 575 (1992)].

${ }^{21}$ A. J. Sievers and S. Takeno, J. Phys. Soc. Jpn. 58, 759 (1989); Phys. Rev. Lett. 61, 970 (1988).

${ }^{22}$ N. G. Vakhitov and A. A. Kolokolov, Izv. Vyssh. Uchebn. Zaved. Radiofiz. 16, 1020 (1973) [Sov. Radiophys. 16, 783 (1975)]; A. A. Kolokolov, ibid. 17, 1332 (1974) [ibid. 17, 1016 (1976)].

${ }^{23}$ P. L. Christiansen, Yu. B. Gaididei, V. K. Mezentsev, S. L. Musher, K. Ф. Rasmussen, J. Juul Rasmussen I. V. Ryzhenkova, and S. K. Turitsyn, Phys. Scr. (to be published).

${ }^{24}$ O. Bang, P. L. Christiansen, F. If, and K. Ø. Rasmussen, Phys. Rev. E 49, 4627 (1994).

${ }^{25}$ D. Möbius and H. Kuhn, Isr. J. Chem. 18, 375 (1979); D. Möbius and H. Kuhn, J. Appl. Phys. 64, 5138 (1988). 